\title{
Increasing the transformer ratio at the Argonne wakefield accelerator
}

\author{
C. Jing, ${ }^{1,2}$ J. G. Power, ${ }^{2}$ M. Conde, ${ }^{2}$ W. Liu, ${ }^{2}$ Z. Yusof, ${ }^{2}$ A. Kanareykin, ${ }^{1}$ and W. Gai ${ }^{2}$ \\ ${ }^{1}$ Euclid Techlabs, LLC, 5900 Harper Road, Solon, Ohio 44139, USA \\ ${ }^{2}$ High Energy Physics Division, Argonne National Laboratory, Argonne, Illinois 60439, USA
}

(Received 22 November 2010; published 16 February 2011)

\begin{abstract}
The transformer ratio is defined as the ratio of the maximum energy gain of the witness bunch to the maximum energy loss experienced by the drive bunch (or a bunch within a multidrive bunch train). This plays an important role in the collinear wakefield acceleration scheme. A high transformer ratio is desirable since it leads to a higher overall efficiency under similar conditions (e.g. the same beam loading, the same structure, etc.). One technique to enhance the transformer ratio beyond the ordinary limit of 2 is to use a ramped bunch train. The first experimental demonstration observed a transformer ratio only marginally above 2 due to the mismatch between the drive microbunch length and the frequency of the accelerating structure [C. Jing, A. Kanareykin, J. Power, M. Conde, Z. Yusof, P. Schoessow, and W. Gai, Phys. Rev. Lett. 98, 144801 (2007)]. Recently, we revisited this experiment with an optimized microbunch length using a UV laser stacking technique at the Argonne Wakefield Accelerator facility and measured a transformer ratio of 3.4. Measurements and data analysis from these experiments are presented in detail.
\end{abstract}

DOI: $10.1103 /$ PhysRevSTAB.14.021302

PACS numbers: 29.20.Ej

\section{INTRODUCTION}

The transformer ratio $(R)$ in a collinear wakefield accelerator is defined as the ratio of the maximum energy gained by the witness bunch to the maximum energy lost by the drive bunch, or, in the case of a bunch train, the maximum energy lost by a bunch in the drive train. It is a figure of merit that helps to characterize the energy transfer efficiency from the drive beam(s) to the trailing witness beam. While $R<2$ for longitudinally symmetric drive beams [1], there are several techniques that can achieve $R>2$ by breaking the symmetry of the drive beam. Examples include an asymmetric longitudinal bunch profile [1], or a bunch train with asymmetrical time structure (equivalent to frequency modulation), or a bunch train with asymmetrical charge structure (equivalent to amplitude modulation) [2]. In this paper, the ramped bunch train (RBT) method was chosen because it is the most mature technology due to the relative ease with which it can be implemented in a photocathode rf gun.

The principle of the RBT technique is shown in Fig. 1. All bunches in the RBT experience the same maximum decelerating field $\left(W_{1}^{-}\right)$while the maximum accelerating field $\left(W_{n}^{+}\right)$behind each bunch increases, thus increasing the transformer ratio $\left(R_{n}=W_{n}^{+} / W_{1}^{-}\right)$with each successive bunch. (This is only strictly true for the center of the bunch but we only wish to illustrate the general concept here.) This is achieved by spacing the bunches at halfinteger multiples of the fundamental wavelength and ramping up their charge according to the ratio 1:3:5:7. The half-integer spacing places the trailing bunch in the accelerating phase of the preceding bunch so that the trailing bunch has its self-wakefield reduced by the accelerating field of the preceding bunch. The charge of trailing bunch is increased until the net decelerating field inside the bunch is equal to the preceding bunch.

During the first joint Euclid-Argonne experiment [3] a RBT of two bunches was sent through a $13.625 \mathrm{GHz}$ dielectric-loaded accelerating structure. The enhancement over the single bunch transformer ratio $\left(R_{1}=W_{1}^{+} / W_{1}^{-}=\right.$ 1.75) was measured to be 1.31 implying $R_{2}=W_{2}^{+} / W_{1}^{-}=$ 2.3. The reason $R$ is only slightly greater than 2 is because
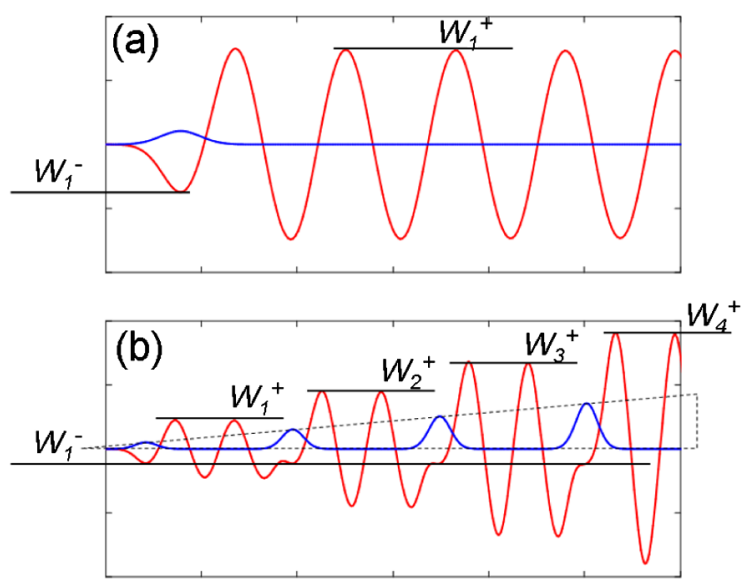

FIG. 1. The ramped bunch train (RBT) method used to generate the transformer ratio, $R>2$. (a) The single bunch wakefield (red) due to a Gaussian drive beam (blue) yields $R_{1}=W_{1}^{+} / W_{1}^{-}=2$. (b) For this structure, a train of four Gaussian drive bunches (blue) with an overall triangular pattern (dotted line) is moving to the left. Bunches are separated by distance $d=2.5 \lambda$ (in general, multiples of $0.5 \lambda$ ), where $\lambda$ is the wavelength of the wakefield. The ratio of the charge is 1:3:5:7; the transformer ratio increases as $R_{n}=W_{n}^{+} / W_{1}^{-}=2 n$. This is only exactly true at the bunch center. 
there was a mismatch between the wavelength of the fundamental mode of the wakefield $(\lambda=22 \mathrm{~mm})$ and the bunch length $\left(\sigma_{z}=2 \mathrm{~mm}\right)$ produced by the Argonne Wakefield Accelerator (AWA) drive gun [4]. To understand the reason for the mismatch we briefly revisit the theory of the RBT.

The theoretical derivation of the single bunch transformer ratio $\left(R_{1}\right)$ and the transformer ratio along the RBT $\left(R_{n}\right)$ are provided in Ref. [3] and we only recap the main results here. (Note, the analysis we present is for a dielectric wakefield accelerator but the conclusion can be generalized to metallic structures and linear plasma wakefield accelerators.) Numerical simulations [5] reveal the dependence of the peak acceleration gradient $\left(W_{1}^{+}\right)$and $R_{1}$ on the bunch length $\sigma_{z}$ (Fig. 2, taken from Ref. [5]). As one would expect, the peak accelerating gradient increases as

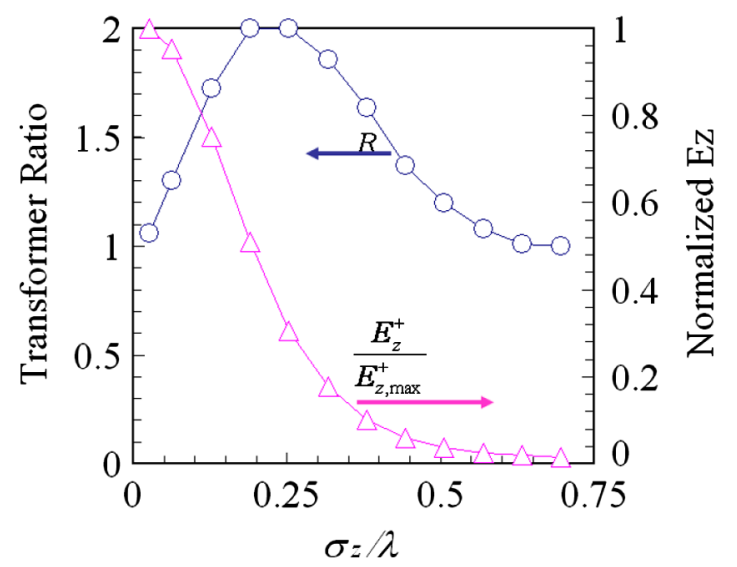

FIG. 2. Transformer ratio for a single bunch. $R$ and peak accelerating field, $E_{z}^{+}$, as a function of the normalized bunch length $\left(\sigma_{z} / \lambda\right)$. The plot is from Ref. [5]. (Single mode operation was assumed in the calculation.) the bunch length decreases, but the behavior of $R_{1}$ as a function of $\sigma_{z} / \lambda$ (where $\lambda$ represents the wavelength of the fundamental accelerating mode) is more complicated than the monotonically decreasing behavior of $W^{+} . R$ is peaked near $\sigma_{z}=\lambda / 4$, while dropping off towards 1 for bunch lengths on either side of $\lambda / 4$. To understand how $\sigma_{z}$ effects the transformer ratio enhancement $\left(R_{n}: R_{n-1}\right)$ numerical simulations were performed for the previous experiment [3] [13.625 GHz dielectric-loaded accelerating (DLA) structure, $\sigma=2 \mathrm{~mm}$ ]. Figure 3(a) shows that there is no significant increase for the transformer ratio after the second drive bunch.

When planning the experiment of this paper we had two options by which we could improve the $\sigma_{z} / \lambda$ ratio over the previous experiment where $\sigma_{z} / \lambda \sim 1 / 11$. We could either increase the AWA drive bunch length to match the frequency of the existing $13.625 \mathrm{GHz}$ DLA structure or fabricate a new DLA structure with its frequency tuned $\lambda \sim 4 \sigma_{z}$. We chose to increase the drive bunch length and use the previously developed and tested $13.625 \mathrm{GHz}$ structure. The AWA drive bunch length was increased with the newly implemented method based on a UV laser pulse stacking technique [6]. Two crystals are used to stretch the AWA laser pulse from 8 to $\sim 24$ ps satisfying the $13.65 \mathrm{GHz}$ structure requirements, which extends the bunch length to $2.7 \mathrm{~mm} \mathrm{rms}$, slightly less than the ideal quarter wavelength value due to rf compression effects in the photoinjector. The exact bunch length chosen was a trade off between the strength of the wakefield and the single bunch transformer ratio. A shorter bunch will excite a larger wakefield, thus making it easier to measure the acceleration of the witness bunch in the experiment, but decrease the transformer ratio.

In Sec. II we discuss the UV laser stacking technique and laser measurement results. Then we present the details of the latest experiment of transformer ratio enhancement
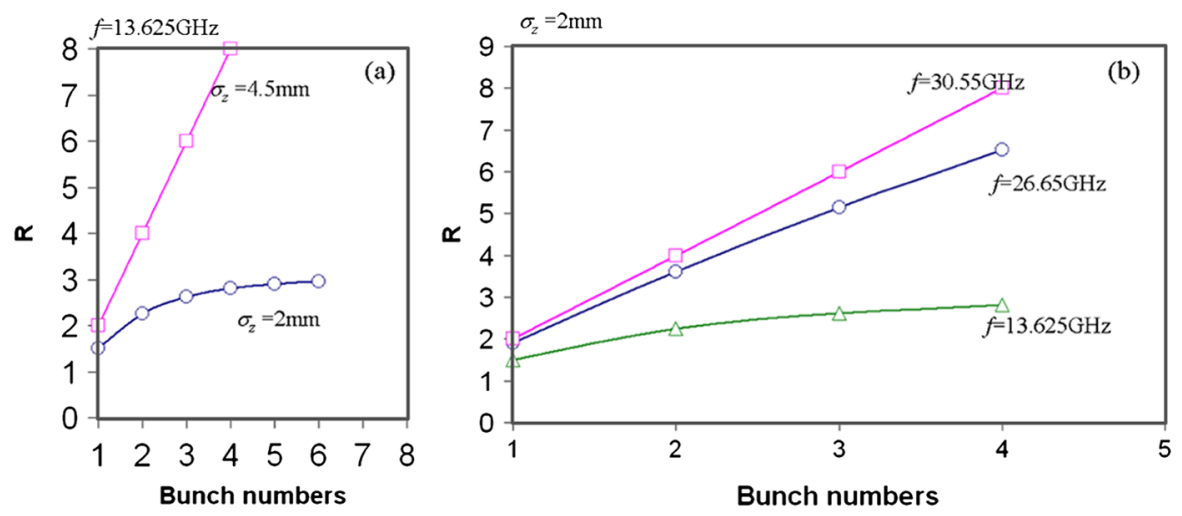

FIG. 3. Transformer ratio for an RBT. $R$ as a function of bunch length and number of bunches for the dielectric wakefield accelerator used in the experiment. (a) $R$ is enhanced in a $13.625 \mathrm{GHz}$ dielectric-loaded accelerator when driven by a longer bunch length $\left(\sigma_{z}=\right.$ $4.5 \mathrm{~mm}$, representative of the old AWA drive gun) compared to a shorter bunch ( $\sigma_{z}=2 \mathrm{~mm}$, the current AWA drive gun). (b) For the current AWA facility, $\sigma_{z}=2 \mathrm{~mm}$ and $R$ can be increased by choosing the correct structure operating frequency. (Note that calculations are based on the single mode wakefield excitation. Shorter bunch lengths may lead to higher order mode excitation inside the structure which will result in slightly different values.) 
in Sec. III, including the experimental results and simulations. Finally, in Sec. IV we summarize the pros and cons of the RBT technique based on experiences gained from the two experiments.

\section{ELECTRON BUNCH LENGTHENING TECHNIQUE: UV LASER STACKING}

The characteristics of the electron bunch emitted from an rf photocathode gun are directly linked to the distribution of the laser pulse striking the photocathode. Therefore, the ability to control the transverse and temporal (longitudinal) laser distribution allows one to create a variety of useful electron bunch profiles. Two methods of longitudinal pulse shaping have been used for rf photocathode guns. The first, spectral masking, is a frequency domain technique that manipulates light in the Fourier transform plane to produce the flattop pulse shape [7]. If this technique is applied in the IR, the pulse shape tends not to be preserved after amplification and harmonic generation. On the other hand, if the spectral masking is applied in the UV the insertion losses can be very large. The second, temporal pulse stacking, directly stacks longitudinally Gaussian pulses into an approximate flattop and was previously accomplished using a combination of half-wave plates and beam-splitting cube polarizers [8]. However, this system is difficult to adjust and align in practice.

In this section, we show how to create the desired longitudinally flattop (or elongated Gaussian) laser pulse shape with birefringent crystals $[9,10]$. The basic idea is to use a series of birefringent crystals to divide the input pulse into a stack of Gaussian pulses and then recombine them. We begin by describing in detail the specific case of four stacked Gaussians. A sketch of how to use two birefringent crystals to create four stacked Gaussian pulses, approximating the flattop, is shown in Fig. 4. The input Gaussian pulse is linearly polarized in the vertical direction while the optical axis of the first crystal (the $14 \mathrm{~mm}$ long crystal in Fig. 4) is tilted at a $45^{\circ}$ angle relative to the vertical. For $\alpha$ - $\mathrm{BBO}\left(\mathrm{BaB}_{2} \mathrm{O}_{4}\right.$, a negative uniaxial crystal), the $e$ ray (or the component of the input pulse that is parallel to the optical axis), will move ahead of the $o$ ray (the component of the input pulse that is perpendicular to the optic axis) by the amount, $\Delta t_{1}$, for a crystal length $L_{1}$. In this case $\left(n_{e}<\right.$ $n_{o}$, where $n_{o}$ and $n_{e}$ are the index of refraction of the $o$ ray and the $e$ ray), the extraordinary axis is the fast axis. The $45^{\circ}$ orientation creates an equal intensity $e$ ray and $o$ ray.

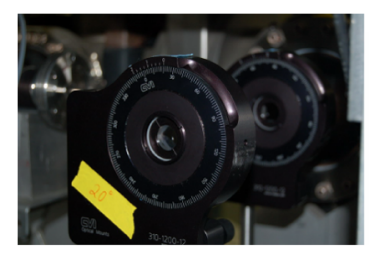

(a)

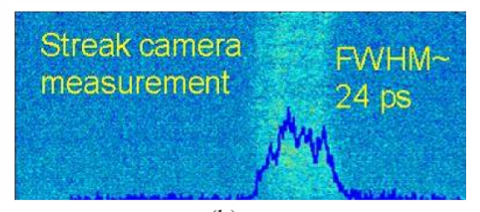

(b)
FIG. 5. Laser pulse stacking characterization. (a) The crystal setup used during the experiment and (b) streak camera measurement of the temporal laser profile after the crystals.

(Notice that the relative intensity between the two rays can be controlled by a simple rotation of the optical axis which leaves open the possibility of triangular shaped pulse generation.) The two (intermediate) pulses emerging from the first crystal are now themselves oriented at $45^{\circ}$ to the vertical. The next crystal, a 7-mm long crystal, has its optical axis oriented in the same direction as the input pulse; i.e., in the vertical. When the two intermediate pulses pass through the second crystal, they are each further divided into two more pulses separated by $\Delta t_{2}$ with crystal length $L_{2}$, thus producing the four output pulses. Streak camera measurement of the temporal laser profile after the crystals is shown in Fig. 5. It indicates that the pulse shape is closer to a "fat" Gaussian than to a flattop pulse due to the imperfections in the technique, including rotation angles and insertion loss variation of the crystals. The electron bunch length resulted from the lengthened laser pulse was simulated in PARMELA. Simulation results show the bunch length was increased from 1.5 to $2.7 \mathrm{~mm}$ (rms), and this value can be maintained over a large charge range of bunch charges, 1 to $15 \mathrm{nC}$.

In the example just considered, an input pulse of intensity $I_{o}$, incident on a pulse stacker of two crystals, will produce four output pulses of intensity $I_{o} / 4$, less insertion losses. In general, it is easy to see that $N$ crystals will generate $2 N$ output pulses of intensity $I_{o} / 2 N$, less insertion losses. Therefore, the number of pulses in the stack is limited by the transparency of the crystals and the amount of laser energy needed for the application.

\section{EXPERIMENT TO ENHANCE TRANSFORM RATIO}

A single UV laser pulse from the AWA laser system is used to generate both the drive RBT and the witness bunch which is used as the main probe to measure the wakefield
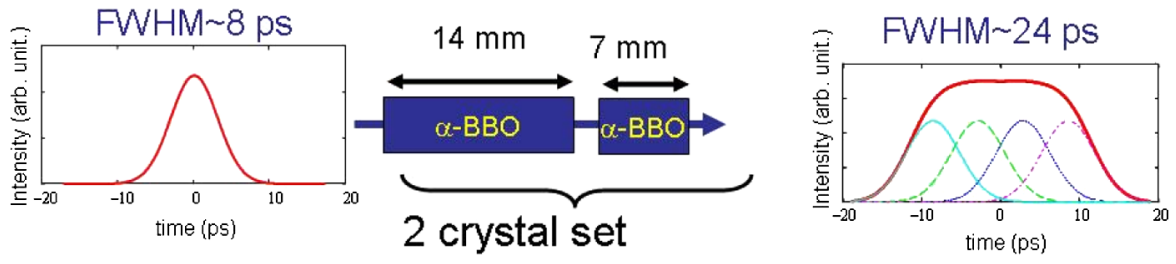

FIG. 4. Laser pulse stacking principle used during the experiment. 
created by the drive RBT. The UV laser pulse is first divided by a $90 \% \_10 \%$ laser beam splitter with the lower energy portion being used to generate the witness beam and the higher portion for the drive RBT. The witness laser pulse is sent directly to the photocathode, thus remaining short and allowing for more accurate measurement of the drive wakefield. An optical delay line on a remotely controlled translation stage was used the control the witness bunch delay relative to the drive bunch train. For the drive RBT, an intensity ramped laser pulse train is formed with laser splitters, mirrors, and neutral density filters (similar to that in the previous experiment [3]) and transported to the photocathode of the AWA $1.3 \mathrm{GHz}$ rf photoinjector to produce the drive RBT with the required charge distribution and intrabunch spacing. The final step is to send the drive laser pulse train through the laser stacking crystals before the injection to the gun to increase the microbunch length of each drive bunch.

The experiment of this paper is similar to the previous one (e.g. conducted in the same $15 \mathrm{MeV}$ AWA beam line) but was improved by increasing the drive bunch length while keeping the witness bunch short (as mentioned above) and by improving the data-taking methods. In the new setup, an independently controlled shutter for each bunch of the RBT's was available. The shutters were remotely controlled and incorporated into the frame grabber controls, which allowed us to quickly acquire the energy spectrometer data in various combinations of drive bunches and witness bunch. For example, we acquired the witness bunch alone, the witness behind one drive bunch, or the witness behind two drive bunches, etc. The charge ratio and bunch spacing are two critical parameters that must be controlled for the transformer ratio to be enhanced. In the previous experiment, the charge information was obtained with two integrating current transformers (ICTs) located before and after the $13.625 \mathrm{GHz}$ dielectric-loaded accelerator. However, the ICT does not have a fast enough response time to distinguish individual bunches in the train (the bunch spacing is $\sim 769 \mathrm{ps}$ or $\sim 23 \mathrm{~cm}$ for the AWA beam line). Thus, while the total charge can be accurately measured using an ICT, the measurement of the charge ratio along the bunch train is difficult. To overcome this difficulty a field probe (a pin probe at the end of coax cable) was placed near a ceramic break in the beam line to monitor the field from the passing electron bunches, allowing us to measure the charge ratio along the train. The ceramic DC break has a very low $Q$ which allowed the signal from the leading bunch to decay significantly before the signal from the following bunch arrives. Using a $16 \mathrm{GHz}$ scope we were able to measure the charge ratio along the ramped bunch train in a single shot (i.e. a single pulse of the accelerator) with reasonable accuracy. Last, a recent upgrade was made to the AWA low level rf system which reduced the short term rf phase jitter down to $1^{\circ}$, which significantly improved the stability of the measurements.
A two-drive-bunch RBT was used in the first phase of the experiment and a four-bunch RBT in the second phase. The measured charge ratio $Q 2 / Q 1=2.7$ was based on several different approaches we will call: (i) ICT average, (ii) pin probe single shot, and (iii) ICT with laser intensity correction (ICT signal was normalized with the laser intensity which was monitored simultaneously through a laser diode). Ideally, the charge ratio of 3 would achieve the highest transformer ratio but this requires using the ideal bunch length $\left(\sigma_{z} / \lambda=1 / 4\right)$. However, due to the slightly shorter bunch length used $(2.7 \mathrm{~mm})$, two longitudinal modes were excited in the DLA structure, the $\mathrm{TM}_{01}$ mode at $13.625 \mathrm{GHz}$ and the $\mathrm{TM}_{02}$ mode at $39.4 \mathrm{GHz}$. We note that the amplitude of the second mode is much smaller so it is not a major limitation. This is because the shorter bunch length leads to a higher wakefield, which in turn makes the energy change of the witness bunch easier to detect. Taking into account the E-field attenuation of the two modes $\left(2.5 \mathrm{~dB} / \mathrm{m}\right.$ for the $\mathrm{TM}_{01}$ and $1.2 \mathrm{~dB} / \mathrm{m}$ for the $\mathrm{TM}_{02}$ mode) and a charge ratio of 2.7 we chose a spacing of $23.07 \mathrm{~cm}$ between the two drive bunches to maximize the wakefield transformer ratio; a value of 3.6 was obtained in the simulation (Fig. 6). The witness bunch location is approximately $70 \mathrm{~cm}$ behind the first drive bunch (Fig. 6) because the fourth drive bunch was $69 \mathrm{~cm}(3 \times 23 \mathrm{~cm})$ behind the lead bunch in the four-bunch RBT case. Although the optical delay line of the witness bunch is independent of the drive RBT, the range over which the remotely controllable fine-tuning range is limited. The coarse position of the witness bunch, which is set by moving a translation stage by hand, remained untouched during the entire experiment for convenience.

Figure 7 compares the measured wakefield created by the first drive bunch alone (blue markers) and the second drive bunch alone (red markers), to the simulations. The measurements were made through monitoring energy

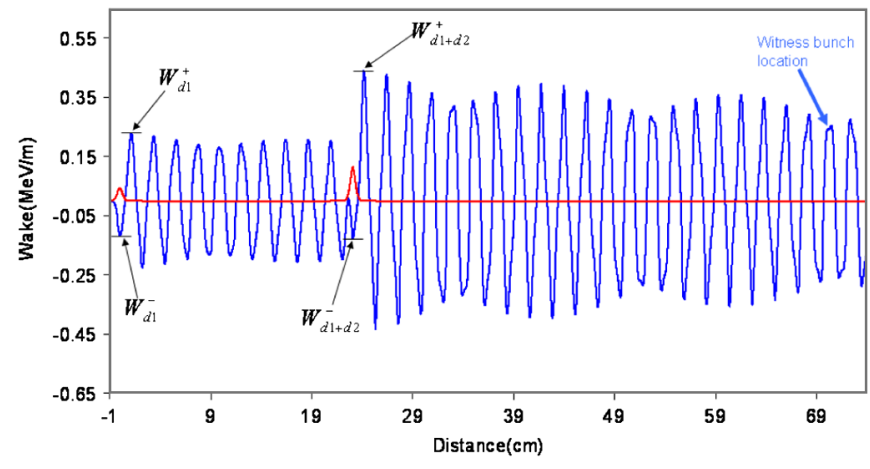

FIG. 6. Simulation of the longitudinal wake potential of the two-bunch RBT. Drive bunches are shown in red and the wake is in blue. In the plot, $W_{d 1}^{+}$and $W_{d 1+d 2}^{+}$represent maximum accelerating fields behind the first and the second drive bunch, respectively, in the two-bunch RBT case. $W_{d 1}^{-}=W_{d 1+d 2}^{-}$represent maximum decelerating fields inside the first and the second drive bunch, respectively. 


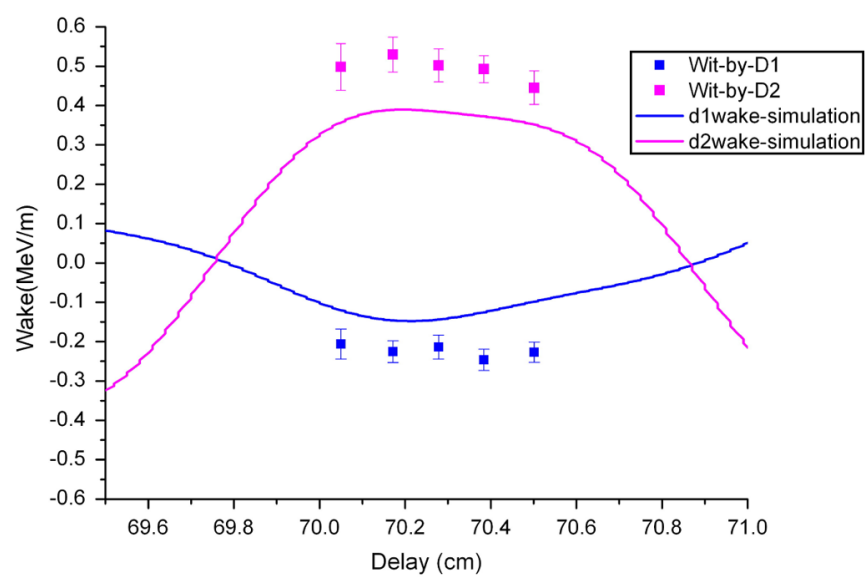

FIG. 7. Wake potential per unit length due to drive bunch \#1 (blue) and \#2 (pink) traversing the $13.625 \mathrm{GHz}$ dielectric accelerator. In the plot, each solid square is a measurement with a mean value of 20 data samples and error bar is the standard deviation.

change of the trailing witness bunch. Note that the single bunch wake generated by adjacent drive bunches is $180^{\circ}$ out of phase due to the half-integer spacing of the RBT method. The simulation results plotted in Fig. 7 show reasonably good agreement with the experimental data.

Measurements of the single bunch transformer ratio, $R_{1}$, were performed first. The maximum decelerating field (Fig. 1, $W_{1}^{-}$) of a single drive bunch was measured by comparing its energy at high charge $\left(Q_{1}\right)$ to its energy at low charge $(\sim$ tens of $\mathrm{pC})$ where the self-wake became negligible. There is a linac tank before the $13.625 \mathrm{GHz}$ DLA structure. But the uncertainty of energy change due to the wakefield of the linac tank is very small compared to the DLA structure because the linac has a very large aperture (verified by simulation with $\mathrm{ABCl}-\mathrm{a}$ wakefield simulation code). The measured peak decelerating wake of the drive bunch after passing through the $0.4 \mathrm{~m}$ long dielectric accelerator was $41 \mathrm{keV} / \mathrm{nC}$ (mean) with a standard deviation $(\sigma)$ of $2.5 \mathrm{keV} / \mathrm{nC}$ (20 samples). The maximum accelerating field (Fig. $1, W 1^{+}$) behind a single drive bunch was measured (with the witness bunch) to be $79.7 \mathrm{keV} / \mathrm{nC}$ (mean) with $\sigma=8.7 \mathrm{keV} / \mathrm{nC}$. Therefore, the transformer ratio of a single drive bunch, $R_{1}$, is 1.94 .

The first measurements of the transformer ratio enhancement $\left(R_{n}: R_{n-1}\right)$ used a two-bunch RBT. Before measuring the maximum wakefield in the accelerating phase from either the first drive bunch alone $\left(W_{d 1}^{+}\right)$or two drive bunches together $\left(W_{d 1+d 2}^{+}\right)$, we carefully tuned the bunch spacing (23.07 $\mathrm{cm}$ between two drive bunches) and charge ratio (2.7) to ensure the second drive bunch was losing the same amount of energy as the lead drive bunch alone (i.e., $W_{d 1}^{-}=W_{d 1+d 2}^{-}$in Fig. 6). The maximum wakefield in the accelerating phase from the first drive bunch was $W_{d 1}^{+}=82.4 \mathrm{keV}(\sigma=15.1 \mathrm{keV})$, and that from both drive bunches together was $W_{d 1+d 2}{ }^{+}=144.6 \mathrm{keV}$

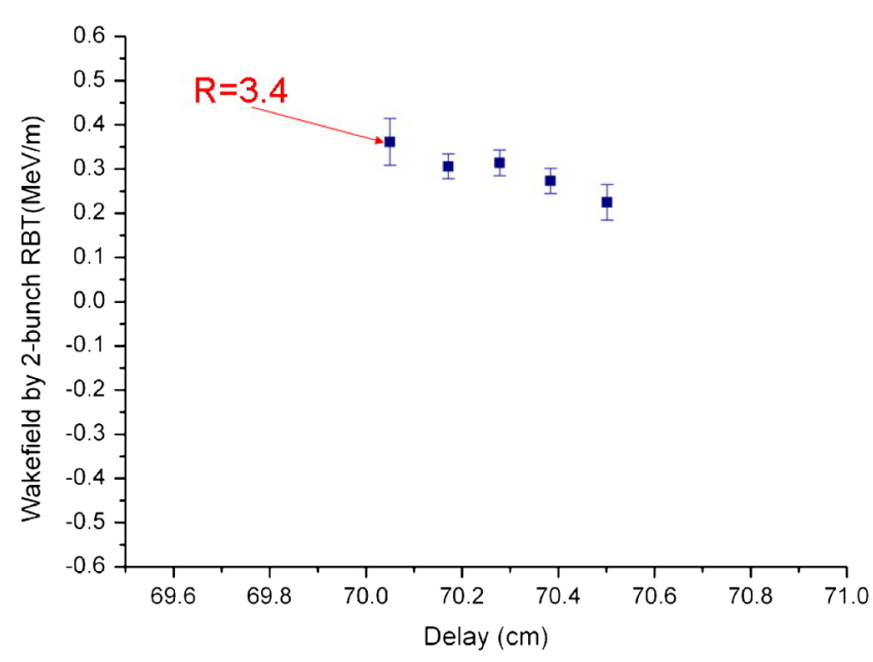

FIG. 8. Measured results of wakefield generated by the twobunch RBT traversing the $13.625 \mathrm{GHz}$ dielectric accelerator. Data points were obtained by measuring the energy change of the witness bunch at various delays. The maximum transformer ratio measured was 3.4 (marked in the plot).

$(\sigma=21 \mathrm{keV})$. This yields a transformer ratio enhancement of $R_{2} / R_{1}=1.75$. Using the measured $R_{1}=1.94$, we have $R_{2}=3.4$. Figure 8 shows the measured results of the wake potential per unit length for the two-bunch ramped bunch train for different delays.

Based on the success of the two-bunch ramped bunch train experiment, we attempted to increase the transformer ratio further with a four-bunch RBT, however, unexpected challenges were encountered during the experiment. The optimal charge ratio of the first and fourth bunches should be $\sim 6.9$, taking into account all factors including the attenuation and existence of $\mathrm{TM}_{02}$ mode. At the same time, the absolute charge for each bunch should be relatively large in order to ensure that the energy change of the witness bunch is detectable. For instance, the large charge discrepancy between the four drive bunches makes it extremely difficult to transmit $100 \%$ of all four drive bunches through the $40 \mathrm{~cm}$ long DLA structure (diameter of the beam channel is $1 \mathrm{~cm}$ ). Another challenge was the inability to obtain the correct charge ratio for all four bunches. In the experiment, a ramped bunch train is formed by the combination of unequal intensity laser beam splitter and neutral density filters (NDF). As pointed out in Ref. [11] it is not possible to obtain the correct charge ratio using NDFs (due to discrete values of the NDF set) in the 4 RBT case.

\section{DISCUSSION}

Two experiments have now been performed using the RBT technique to increase the transformer ratio $R$ above 2 . The value of $R$ equal to 2.3 was achieved in the first experiment and the value of 3.4 has now been obtained in this latest experiment at the AWA facility. The improvement was due to the increased drive bunch length which 
improved the match to the rf wavelength of the DLA. The attempt to extend the experiment to a four-bunch drive train was unsuccessful due the practical considerations. The major challenge was the need to precisely control the charge ratio. A possible advantage of the RBT scheme compared to other schemes to increase the transformer ratio (e.g. a triangular bunch shape) is that laser beam manipulation is easier than electron bunch manipulation. Electron bunch transportation was challenging due to the dramatic difference in charge along the bunch train. For example, in the case of four-bunch RBT, the fourth bunch is 7 times higher in charge than the first one. Since all bunches in the train have the same radius but different charge, the defocusing due to space charge for each bunch in the train is very different which makes it difficult to transport the bunches through the same wakefield device. A method to compensate for this limitation is to scale the radius of all beams so that all beams have the same charge density; this is under development. Another difficulty is that the deflecting force (dipole modes excited by the offaxial beam) increases along the RBT. A higher energy drive beam would mitigate this to some extent, but the overall bunch number in the RBT scheme is limited by these considerations. However, this is not a major drawback since the transformer ratio is enhanced by factor of $2 N$ (where $N$ is the bunch number in the drive train) and a very high $R$ value even with a limited bunch number.

Finally, let us briefly compare the two major techniques used for transformer ratio enhancement: bunch train vs bunch shaping. The comparison is theoretical since the later technique has yet to be experimentally demonstrated. For the comparison, we used the same total bunch length $L$, and only considered single mode operation $\lambda_{0}$ for simplicity. The bunch train distribution has $N$ Gaussian microbunches separated by $\lambda_{0} / 2$, which is the minimum spacing required by the RBT technique [2], so that $L=N \lambda_{0} / 2$. From Ref. [1], for a triangular bunch with the same length,
$L$, yields $R=\pi N / 2$. While from Ref. [3], $R$ for a ramped bunch train increases as $R=2 N$. In other words, the RBT technique increases the transformer ratio slightly faster than the bunch shaping method by a factor of $4 / \pi$. Of course, in an actual wakefield accelerator design, factors other than $R$ also matter. But parameter optimization is a very complicated topic and beyond the scope of our discussion.

\section{ACKNOWLEDGMENTS}

This work was supported by the Department of Energy, Office of High Energy Physics, under Contract No. W-31109-ENG-38.

[1] K. L. Bane, P. Chen, and P. B. Wilson, IEEE Trans. Nucl. Sci. 32, 3524 (1985).

[2] V. M. Tsakanov, Nucl. Instrum. Methods Phys. Res., Sect. A 432, 202 (1999).

[3] C. Jing, A. Kanareykin, J. Power, M. Conde, Z. Yusof, P. Schoessow, and W. Gai, Phys. Rev. Lett. 98, 144801 (2007).

[4] http://www.hep.anl.gov/awa.

[5] J.G. Power and W. Gai, WF-Note-187, http://www .hep.anl.gov/awa/wfnotes/wf187.pdf.

[6] J. G. Power and C. Jing, in Proceedings of the Advanced Accelerator Concept (AIP 1086), Santa Cruz, CA, 2008 (AIP, New York, 2009), pp. 689-695.

[7] A. M. Weiner, Rev. Sci. Instrum. 71, 1929 (2000).

[8] S. Zhou, F. W. Wise, and D. G. Ouzounov, Opt. Lett. 32, 871 (2007).

[9] J. Li, R. Tikhoplava, and A. C. Melissinosa, Nucl. Instrum. Methods Phys. Res., Sect. A 564, 57 (2006).

[10] B. Dromey, M. Zepf, M. Landreman, K. O'Keeffe, T. Robinson, and S. M. Hooker, Appl. Opt. 46, 5142 (2007).

[11] J. G. Power, WF-Note-215, http://www.hep.anl.gov/awa/ wfnotes/wf215.pdf. 